\title{
Universality of QCD Traveling Waves with Running Coupling
}

\author{
Guillaume Beuf $^{1}$, Robi Peschanski ${ }^{1}$ and Sebastian Sapeta ${ }^{2,3}$ \\ 1- Service de Physique Théorique, Orme des Merisiers, CEA/Saclay 91191 Gif-sur-Yvette Cedex, FRANCE \\ 2- Jagiellonian University, Institute of Physics, Ul. Reymonta 4 PL-30-059 Cracow, POLAND. \\ 3- Department of Physics, CERN, Theory Division, CH-1211, Geneva 23, Switzerland.
}

\begin{abstract}
"Geometric scaling", i.e. the dependence of DIS cross-sections on the ratio $Q / Q_{S}$, where $Q_{S}(Y)$ is the rapidity-dependent saturation scale, can be theoretically obtained from universal "traveling wave" solutions of the nonlinear Balitsky-Kovchegov (BK) QCD evolution equation at fixed coupling. We examine the similar mean-field predictions beyond leading-logarithmic order, including running QCD coupling.
\end{abstract}

\section{Motivation}

"Geometric scaling" (GS) is a striking empirical scaling property first observed in deepinelastic (DIS) cross-sections. It consists in the dependence of $\gamma^{*} p$ cross-sections on the ratio $Q / Q_{S}(Y)$, where $\log Q_{S} \propto Y$ is the rapidity-dependent saturation scale. On a theoretical ground, GS can be found as a consequence of saturation effects in QCD, when the density of gluons become large enough to impose unitarity constraints on the scattering amplitude. It has been shown [2] that the QCD evolution with a nonlinear term describing unitarity damping, the Balitsky-Kovchegov (BK) equation, leads to asymptotic "traveling wave" solutions exhibiting the GS property [2]. They are "universal" since they do not depend neither on the initial conditions nor on the precise form of the nonlinear damping terms.

These results were mainly obtained at leading logarithmic order. In the present contribution, we describe how higher orders, in particular incorporating running QCD coupling, influence these predictions. Potential effects may be due to, e.g., nextto-leading (NLL) contributions to the evolution kernel, higher-order resummations schemes, observable dependence, infra-red regularization, position vs. momentum formulation. These aspects and the restauration of universality at high enough rapidity $Y$ has been discussed in Refs.[3], whose re-

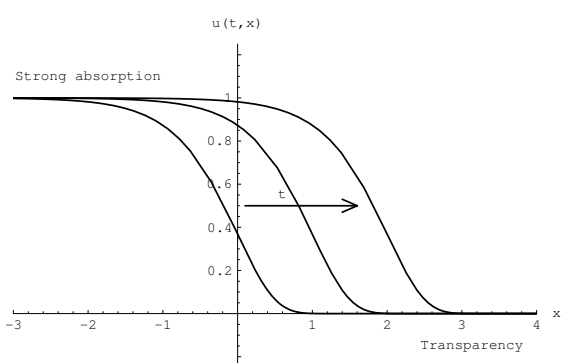

Figure 1: Traveling waves sults are here briefly described. The main difference with the fixed coupling prediction is a new kind of geometrical scaling with $\log Q_{S} \propto \sqrt{Y}$, which appears to be as well verified by data [4] as the original GS property. 


\section{The Balitsky-Kovchegov equation with running coupling}

Before entering the discussion, let us introduce the traveling wave method in the case [2] where the running coupling has been introduced de facto in momentum space. One writes

$$
\left[b \log \left(k^{2} / \Lambda^{2}\right)\right] \partial_{Y} \mathcal{T}=\left\{\chi_{L L}\left(-\partial_{L o g k^{2}}\right)\right\} \mathcal{T}-\mathcal{T}^{2},
$$

where $\mathcal{T}(k, Y)$ is the dipole-target amplitude in momentum space, $\chi_{L L}$ the leading-log QCD kernel and $\left[b \log \left(k^{2} / \Lambda^{2}\right)\right]^{-1} \equiv \alpha\left(k^{2}\right)$, the one-loop QCD running coupling. The asymptotic solutions of the BK equation can be obtained by recognizing the same structure [2] than the traveling wave equation $u(t, x) \rightarrow u(t-v x)$

$$
[x] \partial_{t} u(t, x)=\left\{\partial_{x}^{2}+1\right\} u(t, x)-u^{2}(t, x),
$$

where the traveling-wave/BK "dictionnary" is the following:

Time $=t \sim \sqrt{Y} ;$ Space $=x \sim \log k^{2} ;$ Traveling wave $u(t, x)=u\left(x-v_{c} t\right) \sim \mathcal{T}$.

Using the dictionnary, one thus recognizes the GS property $u\left(x-v_{c} t\right) \sim \mathcal{T}\left(k^{2} / e^{v_{c} \sqrt{Y}}\right)$, with a saturation scale $Q_{S}(Y) \sim e^{v_{c} \sqrt{Y}}$, where $v_{c}$ is the critical wave velocity determined [2] from the linear kernel $\chi_{L L}$.

\section{Traveling waves beyond leading QCD logs}

Let us introduce the general traveling-wave method for the extension beyond QCD leading logarithms. It consists in the following steps:

- Solve the evolution equation restricted to linear terms in terms of a dispersion relation: $u(t, x) \sim \int d \gamma e^{\gamma[x-v(\gamma) t]}$

- Find the critical (minimal) velocity $v_{c}=\min v(\gamma)=v\left(\gamma_{c}\right)$ which is selected by the nonlinear damping independently of its precise form.

- Verify sharp enough initial conditions $\gamma_{0}>\gamma_{c}$, in order for the critical wave form to be selected.

The mathematical properties of such obtained solutions ensure that the corresponding asymptotic solutions are "universal" that is independent from initial conditions, the nonlinear damping terms and from details of the linear kernel away from the critical values. Hence the traveling-wave method defines universality classes from which different equations admit the same asymptotic solutions. One caution is that the range of asymptotics may depend on the singularity structure of the kernel. This may have a phenomenological impact on the possibility of using these solutions in the available experimental range of rapidities. The saddle-point behaves as $\omega_{s} \sim Y^{-1 / 2}$ except near singularities in $\gamma$ of the kernel.

In order to illustrate the method, let us consider the general form of the NLL-extended BK equation, replacing in equation (1), $\chi_{L L}\left(-\partial_{L}\right) \rightarrow \chi_{N L L}\left(-\partial_{L}, \partial_{Y}\right)$, where $L=\log k^{2}$. Introducing the function

$$
X(\gamma, \omega)=\int_{\gamma_{0}}^{\gamma} d \gamma^{\prime} \chi_{N L L}\left(\gamma^{\prime}, \omega\right)
$$

the linear solution reads

$$
\mathcal{T}(L, Y)=\int \frac{d \gamma}{2 \pi i} \mathcal{T}_{0}(\gamma) \exp \left[-\gamma L+\frac{1}{b \omega_{s}}\left(2 X\left(\gamma, \omega_{s}\right)-\omega_{s} \dot{X}\left(\gamma, \omega_{s}\right)\right)\right]
$$


where a dot means $\partial_{\omega}$ and $\omega_{s}$ is given by the saddle-point equation

$$
Y b \omega_{s}^{2}-X\left(\gamma, \omega_{s}\right)+\omega_{s} \dot{X}\left(\gamma, \omega_{s}\right)=0
$$

From the solutions of the saddle-point equation, one can infer [3]:

- For generic kernels beyond leading logs: The kernels may contain singularities up to triple poles due to the NLL contribution. By integration, new single and double-pole singularities appear in $X$ at next leading order. The universality class is still the same but subasymptotics corrections may be large, and thus the critical wave solutions delayed to very large energies.

- For Renormalization-Group improved kernels [5]: The behaviour of the kernels $\chi_{N L L}$ near the singularities are simple poles. This leads only to mild logarithmic singularities in the function $X(\gamma, \omega)$. The net result [3] is that one finds the same universality class as the equation (1), since the $\omega$ dependence in $X$ can be neglected and thus $\chi_{N L L} \rightarrow \chi_{L L}$.

The same approach has been followed for recent QCD formulations of the Balitsky-Kovchegov equation with running coupling constant obtained from quark-loop calculation [6]. It leads to the same conclusion (with the same warning about eventual kernel singularities): the universality class for the BK equations with running coupling is the one defined by Eq.(1).

\section{Geometric Scaling in $\sqrt{Y}$.}

On a phenomenological ground, the main property of solutions corresponding to the universality class of Eq.(1) is the traveling-wave form $u(x, t) \sim u\left(x-v_{c} t\right)=u\left(k^{2} / e^{v_{c} \sqrt{Y}}\right)$ in the asymptotic regime. Assuming a simple relation between that amplitude and the $\gamma^{*} p$ cross-section, one is led to look for geometric scaling of the form $\sigma^{\gamma^{*} p} \sim\left(Q^{2} / e^{v_{c} \sqrt{Y}}\right)$, with $v_{c}=$ cst. In Fig.2, one displays the corresponding data plot [4]. The validity of the scaling property has been quantified using the "Quality Factor" QF method, which allows to determine the adequacy of a given scaling hypothesis with data independently of the form of the scaling curve [4].

One may also use the QF method to evaluate the scheme dependence of the subasymptotic, nonuniversal terms in the theoretical formulae. In this case, the geo-

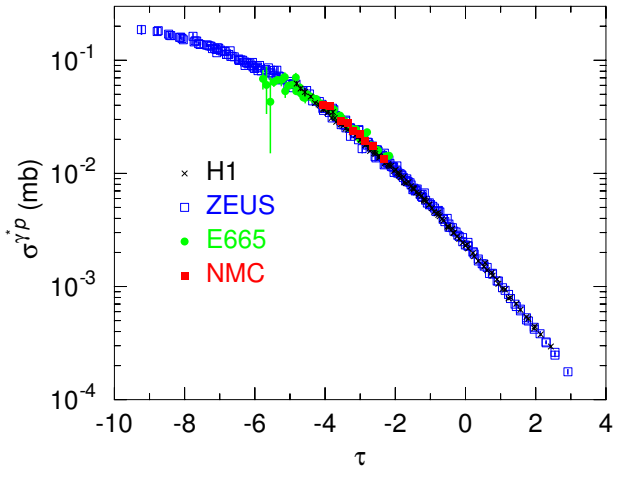

Figure 2: Geometric Scaling in $\sqrt{Y}[4]$ metric scaling prediction is considered in a "strong" version, namely, with the critical parameters (such as $v_{c}$ ) fixed apriori by the theory. In Fig.3 one displays the QF for geometric scaling for different NLL schemes. The top QF is larger than .1 which ensures a good GS property (similar than fig.2). Depending on the resummation scheme $\left(S_{3}, S_{4}, C C S\right.$, see [5]), $\left|Y_{0}\right|$ gives the typical strength of the non-universal terms. The $\mathrm{S} 4$ scheme seems to reach GS sooner (at smaller $\left|Y_{0}\right|$ ). 


\section{Conclusions}

Let us give the main results of our analysis:

- Mean-field saturation beyond leadinglogs: The modified Balitsky-Kovchegov equations including running coupling and higher-order QCD corrections to the linear kernel asymptotically converge to the same traveling-wave solution.

- Characterisation of the universality class: The universality class of these solutions is the BK equation with the leading logarithmic BFKL kernel supplemented by a factorized running coupling whose scale is given by the gluon transverse momentum. Higher order contributions to the kernel will affect the subasymptotic behaviour.

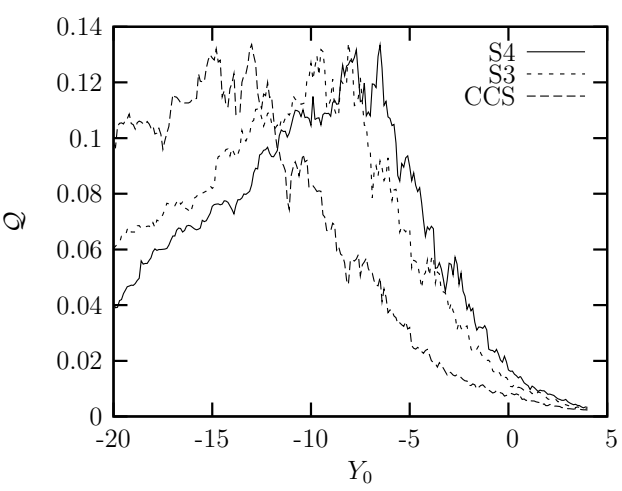

Figure 3: NLL Quality Factors [4]

- Higher-order effects in the kernel: The renormalization-group improved kernels are expected to improve the convergence towards the universal behaviour, spurious singularities being canceled.

- Geometric Scaling: Geometric scaling in $\sqrt{Y}$ is a generic prediction of the universality class of the BK equation with running coupling. It is well borne out by actual data, using the "Quality Factor" method [4] to quantify the validity of the scaling hypothesis without assuming the scaling curve a priori.

- Nonuniversal terms: When using the theoretical "critical" parameters geometrical scaling is verified but requires the introduction of scheme-dependent subasymptotic

Prospects of the present studies are interesting. On the theoretical side, it would be fruitful to investigate the universality properties of QCD equations beyond the mean-field approximation. On the phenomenological side, the problem is still not settled to know whether there is a slow drift towards the universal solutions or whether it exists subasymptotic traveling wave structures, as mathematically [7] or numerically [8] motivated.

\section{References}

[1] Slides: http://indico.cern.ch/contributionDisplay $\cdot$ py? contribId=70\&sessionId=15\& conf Id=9499

[2] S. Munier and R. Peschanski, Phys. Rev. D 69, 034008 (2004).

[3] R. Peschanski and S. Sapeta, Phys. Rev. D 74, 114021 (2006); G. Beuf and R. Peschanski, Phys. Rev. D 75, 114001 (2007).

[4] F. Gelis, R. Peschanski, G. Soyez and L. Schoeffel, Phys. Lett. B 647, 376 (2007).

[5] G. P. Salam, JHEP 07, 019 (1998);

M. Ciafaloni, D. Colferai, and G. P. Salam, Phys. Rev. D60, 114036 (1999).

[6] I. Balitsky, Phys. Rev. D 75, 014001 (2007).

Y. V. Kovchegov and H. Weigert, Nucl. Phys. A 784, 188 (2007).

[7] C. Marquet, R. Peschanski and G. Soyez, Phys. Lett. B 628, 239 (2005)

[8] J. L. Albacete and Y. V. Kovchegov, arXiv:0704.0612 [hep-ph]. 